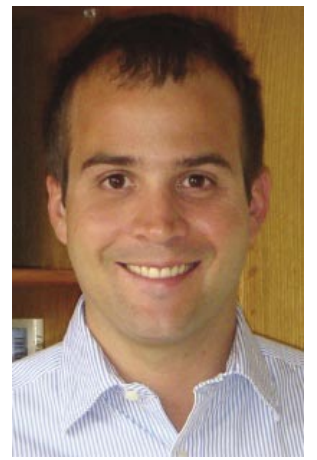

\section{DeWet Swanepoel}

\title{
Newborn hearing screening may predict sudden infant death syndrome
}

\begin{abstract}
Newborn hearing screening has seen tremendous growth in developed countries worldwide, having become the standard of public healthcare, with countries like the USA and UK screening nearly all newborns. Whilst the costs of universal screening for congenital or early onset hearing loss are significant they are offset by the tremendous gains afforded by early intervention in this common (2-6/1000) infant condition. ${ }^{1}$ Infants identified with hearing loss and receiving intervention within the first year of life are able to develop within the range of their normal hearing peers in critical areas of language, speech, cognition and education in stark contrast to late-identified children. ${ }^{2}$
\end{abstract}

Besides these important benefits of newborn hearing screening for infants with hearing loss, a surprising recent report has now linked a specific pattern of results on newborn hearing screening tests to sudden infant death syndrome (SIDS). ${ }^{3}$ This remarkable finding points towards the possibility of identifying children at risk of SIDS through the application of routine newborn hearing screening programmes. SIDS is the most common cause of death in infancy with peak occurrence during the first two to four months of life. Incidence of SIDS varies across socio-economic and even ethnic groups but in developed countries is reported to be at approximately $0.5 / 1000$ occurring slightly more often in males $(60: 40)$. The pathogenesis of the condition to date has, however, remained uncertain. ${ }^{3}$

A recent report by Rubens et al. ${ }^{3}$ investigated newborn hearing screening results retrospectively in a case-controlled study of 31 infants who subsequently died of SIDS matched with surviving controls, based on gender, term versus preterm age and NICU versus well-baby nursery. Hearing screening was conducted with widely used Transient Evoked Otoacoustic Emissions (TEOAE) which measure cochlear outer hair cell integrity across various frequencies. It is a rapid and simple screening test conducted by placing a probe in the newborn ear and presenting broadband click stimuli. Outer hair cell integrity is measured by the mechanical energy of these cochlear structures in response to the auditory stimulation. This response, referred to as an otoacoustic emission, propagates back into the outer ear canal and is recorded by the probe after each click stimulus.

Analysis of results by Rubens et al. ${ }^{3}$ indicated a statistically significant difference $(p<0.05)$ between the amplitude of TEOAE responses in the high frequencies $(2000,3000$, and $4000 \mathrm{~Hz})$ of only the right ears for infants who died of SIDS. The consistently poorer right-sided high frequency results in the experimental group was in contrast to the trend of consistently better right-sided results in the control group, which is in agreement with existing literature pointing to more robust right compared to left ear results. The group of SIDS infants therefore demonstrated a significant reversal of expected TEOAE results for the right ear which can easily be identified by analyzing newborn hearing screening results.

The authors provide important hypotheses for underlying pathogeneses which may lead to this pattern of findings. A perinatal inner ear insult is suggested to be associated with the cochlear findings and the neurological differences associated with SIDS. Placental transfusion differences are highlighted to play an important role, with excessively high pressure posed as a reason for an inner ear insult which may cause a predisposition to SIDS. This is supported by citing reports of lower 1-min Apgar scores for SIDS, ${ }^{4,5}$ increased incidence of SIDS with labours lasting longer than 16 hours ${ }^{5}$ with prima gravida pregnancies 6 and with a second delivered twi ${ }^{7}$. The right ear may be primarily affected due to the preferentially directed blood supply to the right compared to left side which may lead to higher pressures directed to the right. The measured damage in right ear outer hair cell integrity may be representative of further vestibular organ injury, since the inner ear is a membranous labyrinth including the cochlear and vestibular organs. Stimulation of the vestibular system has been demonstrated to initiate the recommencement of respiration from apnoea, and damages to this system may explain apnoea as a common final pathway in SIDS. ${ }^{8,9}$

The possibility of identifying infants at risk for SIDS through a simple screening technique may be an important breakthrough towards implementing preventative measures to avoid a critical incident. This advance towards prevention of a life-threatening condition may provide the necessary momentum for elevating the case for newborn hearing screening as an important healthcare priority, not only in the developed world but also in developing countries. Unfortunately, until now, very few newborn hearing screening programmes have been implemented in South Africa despite the compelling arguments for the quality of life and long-term economic benefits of early intervention for the hearing impaired. In healthcare systems dominated by life-threatening conditions and infectious diseases, chronic non-communicable conditions, especially childhood hearing loss due to its silent nature, have been marginalized for too long ${ }^{1}$. The association of newborn hearing screening with the detection of infants at risk of a fatal condition may therefore serve not only as a breakthrough in saving lives, but may also finally serve to give early intervention for childhood hearing loss in South Africa the imperative it deserves.

DeWet Swanepoel, PhD

Senior Lecturer

Department of Communication Pathology

University of Pretoria

Correspondence to: Dr De Wet Swanepoel, email: dewet.swanepoel@up.ac.za

\section{References}

1. Olusanya BO. Addressing the global neglect of childhood hearing impairment in developing countries. PLOS Med 2007;4(4):e74

2. Moeler PM. Early intervention and language development in children who are deaf and hard of hearing. Pediatrics 2000;106:e43

3. Rubens DD, Vohr BR, Tucker R, O'Neil CA, Chung W. Newborn oto-acoustic emission hearing screening tests: preliminary evidence for a marker of susceptibility to SIDS. Early Hum Dev 2007; doi:10.1016/j.earlhumdev.2007.06.001

4. Buck GM, Michalek AM, Kramer AA, Batt RE. Labor and delivery events and risk of SIDS. Am J Epidemiol 1991;133:900-6.

5. Rhintahaka P. Sudden infant death syndrome in Finland in 1969-1980. Helsinki: Natl Board health; 1985. p. 3.

6. Spiers PS, Lohmann R, Guntheroth WG. Birth order and risk of SIDS: is the true relationship negative? J Paediatr Child Health 1993;29:215-8.

7. Standfast SJ, Jereb S, Aliferis D. Janerick DT. Epidemiology of SIDS in upstate New York. In SIDS, Tildon et al.; 1983. p. 59-75.

8. Harper RM. Autonomic control during sleep and risk for sudden death in infancy. Arch Ital Biol 2001;139(3):185-94.

9. Harper RM, Woo MA, Alger JR. Visualization of sleep influences on cerebellar and brainstem cardiac and respiratory control mechanisms. Brain Res Bull 2000;53(1): 125-31. 